\title{
Late time quantum chaos of pure states in random matrices and in the Sachdev-Ye-Kitaev model
}

\author{
Tokiro Numasawa* \\ Department of Physics, McGill University, Montréal, Québec H3A 2T8, Canada \\ and Department of Physics, Graduate School of Science, Osaka university, Toyonaka 560-0043, Japan
}

(Received 18 January 2019; published 17 December 2019)

\begin{abstract}
We study the return amplitude, which is the overlap between the initial state and the time-evolved state, in the Sachdev-Ye-Kitaev (SYK) model. Initial states are taken to be product states in a spin basis. We numerically study the return amplitude by exactly diagonalizing the Hamiltonian. We also derive the analytic expression for the return amplitude in random matrix theory. The SYK results agree with the random matrix expectation. We also study the time evolution under the different Hamiltonian that is originally proposed to describe the traversable wormholes in projected black holes in the context of holography. The time evolution now depends on the choice of initial product states. The results are again explained by random matrix theory. In the symplectic ensemble cases, we observe an interesting pattern of the return amplitude in which they show the second dip, ramp, and plateaulike behavior.
\end{abstract}

DOI: 10.1103/PhysRevD.100.126017

\section{INTRODUCTION}

The Sachdev-Ye-Kitaev (SYK) model [1,2] is an interesting model. This model is solvable at large $N[3,4]$ but is maximally chaotic [2,5], shows random matrix behaviors $[6,7]$, and shares the same sector with two-dimensional dilaton gravity [8-11].

We study the time dependence of pure states in the SYK model. Here, we consider a special class of pure states that were first considered in Refs. $[12,13]$ and studied further in Refs. [14-16]. They are simultaneous eigenstates of spin operators that are constructed from Majorana fermions. One interesting physical interpretation of these states is that they are states after projection measurements of maximally entangled states by these spin operators $[12,17]$. In this context, we can interpret our setup as time evolution after projection measurements. We expect that the time evolution starts flipping these spins under the SYK Hamiltonian, and we get more general superpositions of these product states. In this paper, we consider the return amplitude, which is the square of the fidelity and used in the similar setup in conformal field theory $[18,19]$. We can also consider the time evolution under deformed Hamiltonians after the measurements. Here, we consider a deformation proposed by Ref. [12]. They are interpreted as a traversable wormhole

\footnotetext{
*tokiro.numasawa@physics.mcgill.ca
}

Published by the American Physical Society under the terms of the Creative Commons Attribution 4.0 International license. Further distribution of this work must maintain attribution to the author(s) and the published article's title, journal citation, and DOI. Funded by SCOAP . protocol to see the insides of black holes $[12,20]$. This deformed Hamiltonian can also be seen as a deformation of an integrable Hamiltonian with degenerate spectrum by the chaotic SYK Hamiltonian.

\section{SYK MODEL}

In the SYK model, we consider even $N$ Majorana fermions $\psi_{i}$ that are obeyed by the anticommutation relation $\left\{\psi_{i}, \psi_{j}\right\}=\delta_{i j}$. The Hamiltonian of the SYK model with $q$ body interactions is given by

$$
H_{\mathrm{SYK}}=i^{\frac{q}{2}} \sum_{a_{1}<a_{2}<\cdots<a_{q}} J_{a_{1} \cdots a_{q}} \psi_{a_{1}} \cdots \psi_{a_{q}} .
$$

Here, $J_{a_{1} \cdots a_{q}}$ are random couplings with mean $\left\langle J_{a_{1} \cdots a_{q}}\right\rangle_{J}=0$ and variance $\left\langle J_{a_{1} \cdots a_{q}}^{2}\right\rangle_{J}=\frac{J^{2}(q-1) !}{N^{q-1}}$. The $q=4$ model is the original SYK model that we mainly focus on in this paper. This system has two important symmetries [6,21]. The first one is the antiunitary symmetry $\mathcal{T}$. This symmetry satisfies $\mathcal{T} \psi_{a} \mathcal{T}^{-1}=\psi_{a}$. The SYK Hamiltonian (1) is invariant under $\mathcal{T}$ when $q=0(\bmod 4)$. The other important symmetry is the modulo 2 fermion number operator $(-1)^{F}$ with $\left((-1)^{F}\right)^{2}=1$. These symmetries can have global anomalies depending on $N(\bmod 8)$ [21]. These anomalies are the origin to realize all of Gaussian unitary (GUE), orthogonal (GOE), and symplectic (GSE) ensembles in the SYK model [6]. Here, we summarize the results $[6,22]$ in Table I.

Now, we consider the return amplitude. The return amplitude is 
TABLE I. Symmetry property in the SYK model.

\begin{tabular}{lcccc}
\hline \hline$N(\bmod 8)$ & $\mathcal{T}^{2}$ & $\mathcal{T}(-1)^{F}=a(-1)^{F} \mathcal{T}$ & Statistics & Degeneracy \\
\hline$N=0$ & +1 & $a=+1$ & GOE & 1 \\
$N=2$ & +1 & $a=-1$ & GUE & 2 \\
$N=4$ & -1 & $a=+1$ & GSE & 2 \\
$N=6$ & -1 & $a=-1$ & GUE & 2 \\
\hline \hline
\end{tabular}

$$
g_{p}(t)=\left|\left\langle\psi_{0}\left|e^{-i H t}\right| \psi_{0}\right\rangle\right|^{2} .
$$

Here, $\left|\psi_{0}\right\rangle$ is a initial state, and $H$ is the Hamiltonian of the system. This definition is also applicable to any quantum systems. We take the square of $\left|\left\langle\psi_{0}\left|e^{-i H t}\right| \psi_{0}\right\rangle\right|$, which is different from the definition in Refs. $[18,19]$. Our choice makes the relation to the so-called spectral form factor clear, which is studied extensively in the field of quantum chaos and also recently in holography [7,23-26].

\section{RETURN AMPLITUDE IN RANDOM MATRICES AND IN THE SYK MODEL}

Because we expect that the late time behavior of the SYK model is governed by random matrices [7], it is good to study first the return amplitude in random matrix theory. Now, we want to compute $\left\langle\left|\left\langle\psi_{0}\left|e^{-i H t}\right| \psi_{0}\right\rangle\right|^{2}\right\rangle_{\mathrm{GUE}}=$ $\int d H e^{-\frac{L}{2} \operatorname{Tr} H^{2}}\left|\left\langle\psi_{0}\left|e^{-i H t}\right| \psi_{0}\right\rangle\right|^{2}$, where $d H$ is the Haar measure on the space of $L \times L$ Hermitian matrices. We can compute this in random matrix theory using the Haar integrals. The results are

$$
\left\langle\left|\left\langle\psi_{0}\left|e^{-i H t}\right| \psi_{0}\right\rangle\right|^{2}\right\rangle_{\mathrm{GUE}}=\frac{1}{L(L+1)}\left(\langle g(t)\rangle_{\mathrm{GUE}}+L\right),
$$

where $g(t)=Z(t) Z(t)^{*}$ with $Z(t)=\operatorname{Tr}\left(e^{-i H t}\right)$. This $g(t)$ is the spectral form factor, which diagnoses the energy-level correlations in chaotic systems. We also find that this relation also holds in the GSE by replacing the average with \langle\rangle$_{G S E}$, using the Haar integral $[27,28]$. This formula that relates the spectral form factor to the return amplitude in random matrices in the GUE and GSE is our first main result on the random matrices side. ${ }^{1}$ We put the derivation of this formula in Appendix B. In the GOE, the return amplitude depends on the initial state $\left|\psi_{0}\right\rangle$. Equation (3) means that the return amplitude is given essentially by the constant $L$ shift of the spectral form factor. When $t=0$, the spectral form factor is simply given by the square of the dimension of the Hilbert space $g(0)=L^{2}$. Under the time evolution, $\langle g(t)\rangle_{\mathrm{GUE}}$ decreases and hits the minimal value. These regimes are called the slope and the dip [7]. Then, $\langle g(t)\rangle_{\mathrm{GUE}}$ increases linearly. This linear growth is called the ramp [7], and this reflects the long-range eigenvalue correlations in chaotic systems. Finally, $\langle g(t)\rangle_{\mathrm{GUE}}$ saturate

\footnotetext{
${ }^{1}$ The return amplitude in the GOE is also studied in Ref. [29].
}

the late time value $\langle g(\infty)\rangle_{\mathrm{GUE}}=L$ in sufficiently late time. This is called the plateau [7]. The plateau value generically coincides with the infinite time average,

$$
\lim _{T \rightarrow \infty} \frac{1}{T} \int_{0}^{T} d t \sum_{m, n} e^{-i\left(E_{m}-E_{n}\right) t}=\sum_{E} N_{E},
$$

where $N_{E}$ is the degeneracy of each energy level $E$. This plateau value is much smaller than the initial value $g(0)=L^{2}$ but still bigger than $\mathcal{O}(1)$. The relation (3) between the return amplitude $g_{p}(t)$ and the spectral form factor $g(t)$ says that the return amplitude also shows the slope, the dip, the ramp, and the plateau. The plateau value for the return amplitude is given by $\left\langle g_{p}(t)\right\rangle_{\mathrm{GUE}}=$ $2 /(L+1)$, which is also much smaller than the initial value $g_{p}(0)=1$.

The spectral form factor has a finite temperature generalization $g(t ; \beta)=\operatorname{Tr}\left(e^{-\beta H-i H t}\right) \operatorname{Tr}\left(e^{-\beta H+i H t}\right)$. For a finite temperature analog of the return amplitude, we consider

$$
g_{p}(t ; \beta)=\left|\left\langle\psi_{0}\left|e^{-\beta H-i H t}\right| \psi_{0}\right\rangle\right|^{2}
$$

We can think of this as the return amplitude with the initial state $e^{-\frac{\beta}{2} H}\left|\psi_{0}\right\rangle$. As is the case with the finite temperature spectral form factor [7], when we take the ensemble average, the annealed disorder $\frac{\left\langle\left|\left\langle\psi_{0}\left|e^{-\beta H-i H t}\right| \psi_{0}\right\rangle\right|^{2}\right\rangle_{E}}{\left.\left\langle\left|\psi_{0}\right| e^{-\beta H} \mid \psi_{0}\right\rangle||^{2}\right\rangle_{E}}$ is not equal to the quenched disorder $\left\langle\frac{\left|\left\langle\psi_{0}\left|e^{-\beta H-i H t}\right| \psi_{0}\right\rangle\right|^{2}}{\left|\left\langle\psi_{0}\left|e^{-\beta H}\right| \psi_{0}\right\rangle\right|^{2}}\right\rangle_{E}$, where \langle\rangle$_{E}$ is an ensemble average. In this paper, we consider the annealed disorder in which the analytic treatment becomes easy $[7,30]$. Another motivation to take the annealed version is its similarity with the unnormalized cylinder amplitude in quantum field theories $[18,19]$. The ensemble average of the finite temperature return amplitude (5) in random matrix theory becomes

$$
\begin{aligned}
& \left\langle\left|\left\langle\psi_{0}\left|e^{-\beta H-i H t}\right| \psi_{0}\right\rangle\right|^{2}\right\rangle_{\mathrm{GUE}} \\
& =\frac{1}{L(L+1)}\left(\langle g(t ; \beta)\rangle_{\mathrm{GUE}}+\langle g(0 ; 2 \beta)\rangle_{\mathrm{GUE}}\right) .
\end{aligned}
$$

This formula that relates the finite temperature spectral form factor to the finite temperature return amplitude in random matrices in the GUE is our second main result in random matrices side. We call the right-hand side of (3) and (6) the shifted spectral form factor.

Now, we consider the return amplitude in the SYK model. The states we consider are defined as follows. First, there are $N / 2$ spin operators in the SYK model defined by $S_{k}=-2 i \psi_{2 k-1} \psi_{2 k}$. These satisfy $S_{k}^{2}=1$, and eigenvalues of $S_{k}$ are given by $s_{k}= \pm 1$. The pure states $\left|B_{s}\right\rangle$ we consider are defined as common eigenstates of these spin operators:

$$
S_{k}\left|B_{s}\right\rangle=s_{k}\left|B_{s}\right\rangle, \quad \text { for } k=1, \ldots, N / 2 .
$$




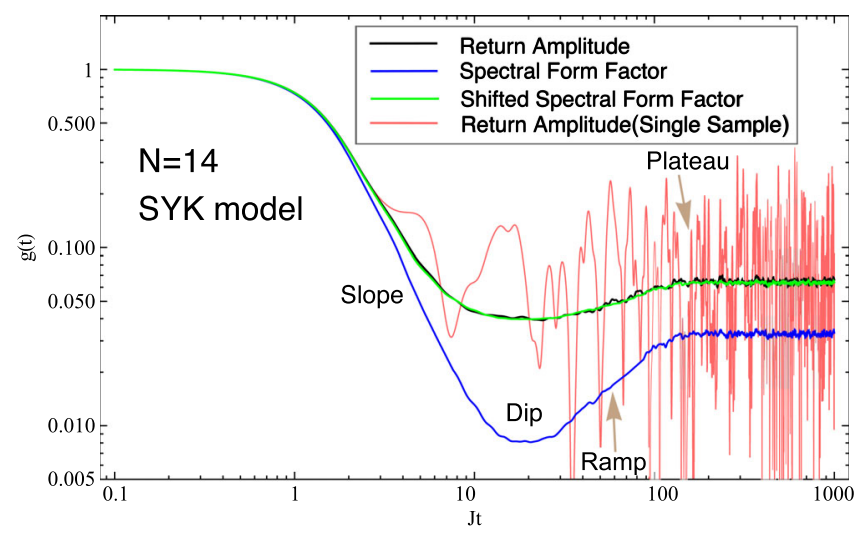

FIG. 1. These are numerical plots for the $N=14 q=4 \mathrm{SYK}$ model. We take the disorder average for 1500 samples except for the single sample case. We put $\beta=1.5$. The return amplitude is defined (9), and we choose the state that satisfies $S_{k}\left|B_{\uparrow \ldots \uparrow}\right\rangle=\left|B_{\uparrow \ldots \uparrow}\right\rangle$. The shifted spectral form factor is the righthand side of (6) for the SYK Hamiltonian. We normalize them so that the initial values become 1 . A single sample of $g_{p}(t)$ shows erratic oscillation around the averaged return amplitude at late time, and it is not self-averaging [32].

This defines $2^{\frac{N}{2}}$ states, one for each choice of the spins $s_{k}$. These states form a basis of the SYK Hilbert spaces. By including the Euclidean evolution in the SYK Hamiltonian, we can produce lower-energy states:

$$
\left|B_{s}(\beta)\right\rangle=e^{-\frac{\beta}{2} H_{\mathrm{SYK}}}\left|B_{s}\right\rangle .
$$

The return amplitude for the SYK model is

$$
g_{p}(t ; \beta)=\left|\left\langle B_{s}\left|e^{-\beta H_{\mathrm{SYK}}-i H_{\mathrm{SYK}} t}\right| B_{s}\right\rangle\right|^{2} .
$$

Currently, we do not have any technique to analytically compute (9) for finite $N$, and we numerically computed the finite temperature return amplitude (9) in the SYK model. The results are plotted in Fig. 1. Clearly, we observe the slope, the dip, the ramp, and the plateau in the return amplitude in the SYK model. The early time decay is almost the same with the spectral form factor. In the large $N$ limit, $\left\langle B_{s}\left|e^{-\beta H_{\mathrm{SYK}}}\right| B_{s}\right\rangle=2^{-\frac{N}{2}} \operatorname{Tr}\left(e^{-\beta H_{\mathrm{SYK}}}\right)+\mathcal{O}\left(1 / N^{q-1}\right)$ for any $\left|B_{s}\right\rangle[12]$ in the leading of $1 / N$ expansion. The early time dependence is captured by the analytic continuation of $\beta \rightarrow \beta+i$ trom the leading term in $1 / N$, and we expect the match between them. On the other hand, in the ramp and the plateau regions, we do not expect that because they are nonperturbative effects in $1 / N$ expansion $[7,31]$. The plot shows that they take different values at late time. The late time behavior is expected to be governed by random matrix theory [7]. We also for the return amplitude. To confirm this, we compare the return amplitude with the shifted spectral form factor (6) in which the ensemble average $\langle g(t ; \beta)\rangle_{\mathrm{GUE}}$ is replaced by the SYK coupling average $\langle g(t ; \beta)\rangle_{J}$. We also restrict the Hamiltonian to the fixed
$(-1)^{F}$ charge sector in the shifted spectral form factor because only that acts on the state $\left|B_{s}\right\rangle$. The plots agree very well, and these results also support the random matrix behavior in the late time in the SYK model.

\section{EVOLUTION AMPLITUDE IN RANDOM MATRICES AND IN THE SYK MODEL}

Equation (3) also means that the initial state $\left|\psi_{0}\right\rangle$ can evolve to other states that are orthogonal to $\left|\psi_{0}\right\rangle$. Let us pick a state $\left|\psi_{1}\right\rangle$ that satisfies $\left\langle\psi_{1} \mid \psi_{0}\right\rangle=0$. In the similar way with the return amplitude, we can calculate the overlap $\left|\left\langle\psi_{1}\left|e^{-i H t}\right| \psi_{0}\right\rangle\right|^{2}$. Here, we call this the evolution amplitude. The evolution amplitude becomes

$\left\langle\left|\left\langle\psi_{1}\left|e^{-i H t}\right| \psi_{0}\right\rangle\right|^{2}\right\rangle_{\mathrm{GUE}}=\frac{1}{L^{2}-1}\left(L-\frac{\langle g(t)\rangle_{\mathrm{GUE}}}{L}\right)$.

This expression for the evolution amplitude in terms of the spectral form factor is our third main result in random matrices side. We put the derivation of this formula in Appendix B. The evolution amplitude increases under the time evolution from 0 , then slightly decreases, and finally saturates the late time value $1 /(L+1)$. Interestingly, this late time value is not equal to the late time value of the return amplitude $g_{p}(\infty)=2 /(L+1)$ but half of that. On the other hand, we get $\int d U|\langle\psi|U| \phi\rangle|^{2}=1 / L$ for any choice of $|\psi\rangle$ and $|\phi\rangle$ for Haar random unitary $U$. This is because the average with $\lim _{T \rightarrow \infty} \int d H e^{-\frac{L}{2} \operatorname{Tr} H^{2}} F\left(e^{-i H t}\right)$ is not equivalent to the Haar random unitary average $\int d U F(U)$ for functions $F$ on the space of $L \times L$ unitary groups [31].

In the GUE case, the spectral form factor at infinite temperature is given by [30]

$\langle g(t)\rangle_{\mathrm{GUE}}=L^{2} \frac{J_{1}(t)^{2}}{t^{2}}-L\left(1-\frac{t}{2 L}\right) \theta(2 L-t)+L$,

where $J_{\alpha}(t)$ is the Bessel function of the first kind and $\theta(t)$ is the Heaviside step function. Together with this expression of the spectral form factor and (3) or (10), we can determine the exact time evolution of the return amplitude and the evolution amplitude in the GUE. We plot (3) and (10) in Fig. 2. Clearly, the return amplitude acquires a larger value than the evolution amplitude at late times. In this sense, the time-evolved state $e^{-i H t}\left|\psi_{0}\right\rangle$ never forgets about the direction of the initial state $\left|\psi_{0}\right\rangle$. This plot also makes it clear that the return amplitude starts to acquire the larger value from the ramp region, which is the signature of quantum chaos.

Now, we consider the evolution amplitude in the SYK model. Especially, we consider the amplitude between $\left|B_{s}\right\rangle$ with different spins:

$$
\left|\left\langle B_{s^{\prime}}\left|e^{-i H_{\mathrm{SYK}} t}\right| B_{s}\right\rangle\right|^{2} .
$$




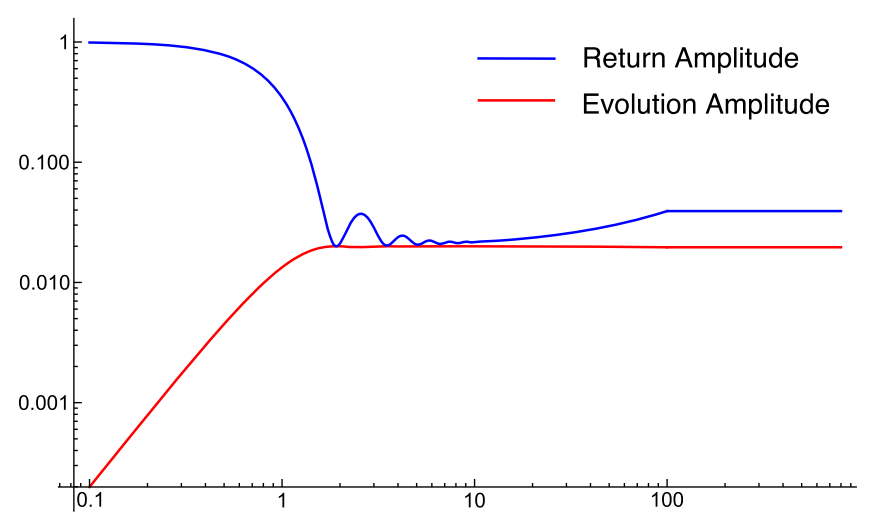

FIG. 2. The plot of the return amplitude and the evolution amplitude in random matrices in the GUE with $L=50$. From this picture, the return amplitude acquires a larger value than the evolution amplitude because of the ramp that is the signal of quantum chaos.
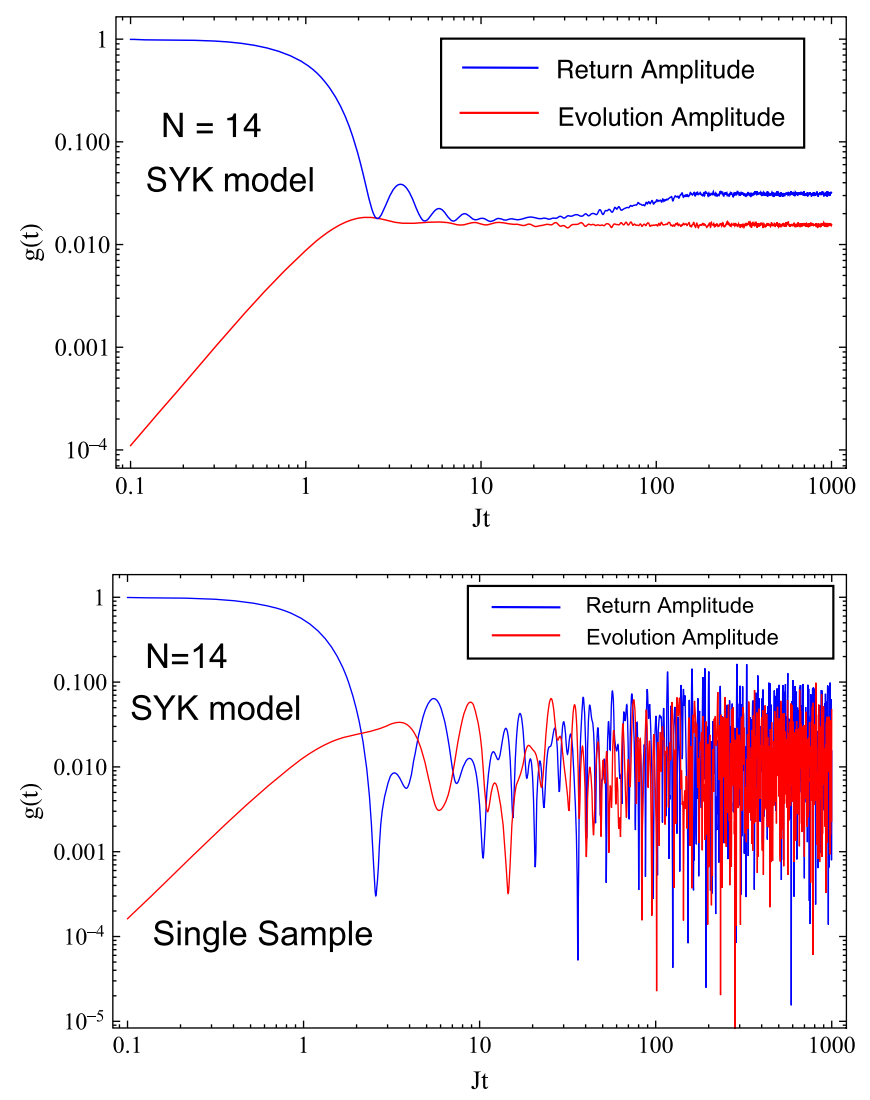

FIG. 3. These are the numerical plots of the return amplitude and the evolution amplitude in the $N=14, q=4 \mathrm{SYK}$ model. For an example of the return amplitude, we consider $\left|\left\langle B_{\uparrow \uparrow \uparrow \uparrow \uparrow \uparrow \uparrow}\left|e^{-i H_{\mathrm{SYK}} t}\right| B_{\uparrow \uparrow \uparrow \uparrow \uparrow \uparrow \uparrow}\right\rangle\right|^{2}$, and for an example of the evolution amplitude, we consider $\left|\left\langle B_{\downarrow \downarrow \uparrow \uparrow \uparrow \uparrow \uparrow}\left|e^{-i H_{\mathrm{SYK}} t}\right| B_{\uparrow \uparrow \uparrow \uparrow \uparrow \uparrow \uparrow \uparrow}\right\rangle\right|^{2}$. Top: We take the average over 1500 samples for both the return amplitude and the evolution amplitude. Bottom: The return amplitude and the evolution amplitude with the single sample.
In Fig. 3, we compare the return amplitude and the evolution amplitude numerically in the SYK model. After the ensemble average, they show that the plateau value in the return amplitude is clearly larger than the evolution amplitude. Even in single realization, the return amplitude looks to take larger value than the evolution amplitude.

\section{DEFORMED HAMILTONIAN}

Next, we consider the following "mass term" Hamiltonian [12]:

$$
H_{M}=-\frac{1}{2} \sum_{k} s_{k} S_{k}=i \sum_{k} s_{k} \psi_{2 k-1} \psi_{2 k} .
$$

This Hamiltonian is diagonalized in the $\left|B_{s}\right\rangle$ state basis. Especially, the unique ground state of this Hamiltonian is given by $\left|B_{s}\right\rangle$ with spin $\left\{s_{k}\right\}$ and energy $E_{0}^{(0)}=-N / 4$. By flipping some spins from the ground state $\left|B_{s}\right\rangle$, we obtain the whole energy eigenstates. The excited state energy levels are given by

$E_{m}^{(0)}=-\frac{N}{4}+m$ with degeneracy $d_{m}=\left(\begin{array}{c}N / 2 \\ m\end{array}\right)$.

There are energy gaps, which are given by $E_{m+1}^{(0)}-$ $E_{m}^{(0)}=1$, between the bands. Now, we consider the Hamiltonian that contains the both the SYK term and (13):

$$
H_{\mathrm{def}}=H_{\mathrm{SYK}}+\mu H_{M}
$$

This Hamiltonian was originally proposed to describe the traversable wormhole after projection measurements [12]. We call this $H_{\text {def }}$ the deformed Hamiltonian. Here, we consider the regime that $\mu$ is large, and we can treat the SYK term as a perturbation. This can be seen as a perturbation of the integrable system with the degenerate spectrum by the chaotic Hamiltonian. ${ }^{2}$ We also concentrate on the infinite temperature cases. Because $\mu$ is large, exact energy levels from $E_{i_{m}}$ with $i_{m}=d_{1}+\cdots+d_{m-1}+1$ to $E_{f_{m}}$ with $f_{m}=d_{1}+\cdots+d_{m}$ localize near $E_{m}^{(0)}$ and form a bandlike structure. By exactly diagonalizing the Hamiltonian, we can study the return amplitude under the deformed Hamiltonian. We show the numerical results in Fig. 4. Here, we explain the results. First, the ground state of $H_{\text {def }}$ is almost given by $\left|B_{s}\right\rangle$ with $m=0$ because the gap between the ground state and the first excited states is $\mu$, which is sufficiently large and suppresses the mixing with other $\left|B_{s}\right\rangle$ states. In this case, the return amplitude does not decay and also shows oscillation at early time.

${ }^{2}$ Other kinds of mass deformations are considered in Refs. [33-35] 


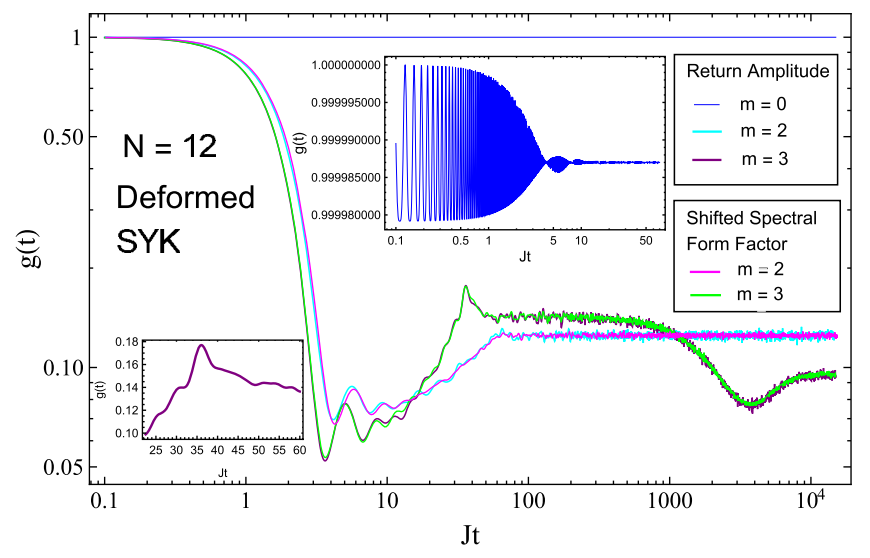

FIG. 4. These are numerical plots for the $N=12 q=4$ deformed SYK model with $\mu=50$ and $s_{k}=1$ in (13) for all $k$. We take the average over 2000 samples. $m$ is the number of flips of spins from the B state from the ground state of $H_{M}$. More explicitly, we choose $\left|B_{\uparrow \uparrow \uparrow \uparrow \uparrow \uparrow \uparrow}\right\rangle$ for $m=0,\left|B_{\downarrow \downarrow \uparrow \uparrow \uparrow \uparrow}\right\rangle$ as an example of $m=2$ cases and $\left|B_{\downarrow \downarrow \downarrow \uparrow \uparrow \uparrow}\right\rangle$ for $m=3$. We also plot the shifted spectral form factor of band $m$, where the spectral form factor restricted on the $m$ th band is $\sum_{i, j=i_{m}}^{f_{m}} e^{-i\left(E_{i}-E_{j}\right) t}$ and the shift (3) is given by $L=d_{m}$. We see the kink around the transition time from the ramp to the plateau in the $m=3$ case, which reflects GSE statistics [7].

The behavior of $\left|B_{s}\right\rangle$ with $m \neq 0$ is described by random matrix theory. According to the perturbation theory of quantum mechanics, the first-order shift of energy levels is determined by the projection of $H_{\mathrm{SYK}}$ onto the degenerate energy levels [36]. Therefore, at early time, the projection of $H_{\mathrm{SYK}}$ on each degenerate energy level determines the time evolution of $\left|B_{s}\right\rangle$. We know the dimension of the projected Hamiltonian from (14). By considering the spectral form factor restricted on the $m$ th band and its shift by $L=d_{m}$, we see the very good agreement with the return amplitude. Because the spectral form factor and the return amplitude depend on the level statistics, next we determine the symmetry class of each band. To see this, we need to know the symmetry properties of $\left|B_{s}\right\rangle$. These are eigenstates of $(-1)^{F}$. The antiunitary $\mathcal{T}$ flips the spin $\mathcal{T} S_{k} \mathcal{T}^{-1}=$ $-S_{k}$. Therefore, we find that $\mathcal{T}\left|B_{s}\right\rangle=e^{i \theta_{s}}\left|B_{-s}\right\rangle$, where $e^{i \theta_{s}}$ is a phase factor and $\left|B_{-s}\right\rangle$ is the state that satisfies $S_{k}\left|B_{-s}\right\rangle=-s_{k}\left|B_{-s}\right\rangle$. From this, we find that for the projection operator onto the flip number $m$ sector $P_{m}=$ $\sum_{\# \text { \#lip }=m}\left|B_{s}\right\rangle\left\langle B_{s}\right|$ satisfies

$$
\mathcal{T} P_{m} \mathcal{T}^{-1}=P_{\frac{N}{2}-m}
$$

This means that $\mathcal{T}$ flips the bands.

Now, we consider the symmetry class based on these properties. First, we consider the $m \neq \frac{N}{4}$ bands. In this case, $H_{m}$ satisfies $\mathcal{T} H_{m} \mathcal{T}^{-1}=H_{\frac{N}{2}-m}$. Therefore, there are no constraints from the symmetry $\mathcal{T}$, and we expect GUE statistics for $H_{m}$. Next, we consider $m=\frac{N}{4}$ th bands, which exist in $N=0,4(\bmod 8)$ cases. Now, $\mathcal{T}$ imposes symmetry constraint $\mathcal{T} H_{\frac{N}{4}} \mathcal{T}^{-1}=H_{\frac{N}{4}}$, and we expect the GOE for $N=0(\bmod 8)$ and GSE for $N=4(\bmod 8)$ on these bands at the first order of perturbation. Except for $m=\frac{N}{4}$ th bands in $N=4(\bmod 8)$, we expect that degeneracies are completely removed by the SYK Hamiltonian perturbation. Because of Kramers's degeneracies of $H_{\frac{N}{4}}$ that originate to the time-reversal anomaly $\mathcal{T}^{2}=-1, m=\frac{N}{4}$ th bands in $N=$ $4(\bmod 8)$ still have two degeneracies at each level at the first-order perturbation. According to the second-order perturbation theory [36], the second-order shift of degenerate spectra is determined by $H_{\frac{N}{4}}^{i}=\mathcal{P}_{i} H_{\mathrm{SYK}} \mathcal{Q}_{\frac{N}{4}} H_{\mathrm{SYK}} \mathcal{P}_{i}$. Here, $\mathcal{Q}_{\frac{N}{4}}=\sum_{m \neq \frac{N}{4}} \frac{P_{m}}{-\frac{N}{4}+m}$ and $\mathcal{P}_{i}=\left|\psi_{i, 1}\right\rangle\left\langle\psi_{i, 1}|+| \psi_{i, 2}\right\rangle\left\langle\psi_{i, 2}\right|$ with two eigenstates of $i$ th degenerate eigenvalues. We choose the basis that satisfies $\mathcal{T}\left|\psi_{i, 1}\right\rangle=\left|\psi_{i, 2}\right\rangle$. From these definitions, we find that $\mathcal{T} \mathcal{P}_{i} \mathcal{T}^{-1}=\mathcal{P}_{i}$ and $\mathcal{T} \mathcal{Q}_{\frac{N}{4}} \mathcal{T}^{-1}=-\mathcal{Q}_{\frac{N}{4}}$. This means $\mathcal{T} H_{\frac{N}{4}}^{i} \mathcal{T}^{-1}=-H_{\frac{N}{4}}^{i}$. By solving this symmetry constraint in the basis with $\mathcal{T}=\left(\begin{array}{cc}0 & -1 \\ 1 & 0\end{array}\right) K$, we obtain

$$
H_{\frac{N}{4}}^{i}=\mathcal{P}_{i} H_{\mathrm{SYK}} \mathcal{Q}_{\frac{N}{4}} H_{\mathrm{SYK}} \mathcal{P}_{i}=\left(\begin{array}{cc}
x & a \\
a^{*} & -x
\end{array}\right),
$$

with a real number $x$ and a complex number $a$. This means that in a generic Hamiltonian the off-diagonal element $a$ enters and the degeneracies are removed. Therefore, the degeneracy is removed at the second order of the perturbation. This difference of the order means that the return amplitude (and the spectral form factor) does not see the true degeneracy at early time. We see numerically in Fig. 4 that they show the first-order degeneracy at the first plateau, but after that, they show the second slop, dip, ramp, and plateau. The second plateau value is smaller than the first plateau value because (4) means that degeneracies give larger plateau values.

To see the level statistics further, we study the distribution of the adjacent gap ratio [6,37-39] for each $m$ th band (see Fig. 5). The adjacent gap ratio is defined as $r_{n}=$ $\frac{E_{n+1}-E_{n}}{E_{n}-E_{n-1}}$ for an ordered spectrum $E_{n-1}<E_{n}<E_{n+1}$. The distribution of the ratio $r_{n}$ in Poisson statistics is

$$
p(r)=\frac{1}{(1+r)^{2}} .
$$

On the other hand, in random matrices, the distribution of the ratio $r_{n}$ becomes [40]

$$
p(r)=\frac{1}{Z_{\beta}} \frac{\left(r+r^{2}\right)^{\beta}}{\left(1+r+r^{2}\right)^{1+\frac{3}{2} \beta}} .
$$

For the GOE, $\beta=1$ and $Z_{\beta}=\frac{27}{8}$. For the GUE, $\beta=2$ and $\frac{4 \pi}{81 \sqrt{3}}$. For the GSE, $\beta=4$ and $Z_{\beta}=\frac{4 \pi}{729} . r \rightarrow 0$ behavior 

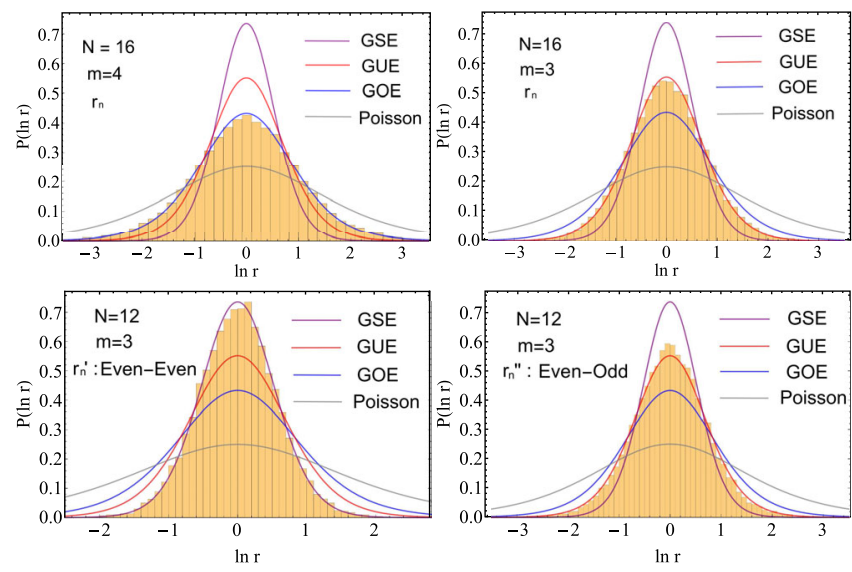

FIG. 5. We plot the probability density $P(\ln r)=p(r) r$ for some examples. The solid lines are the Wigner surmise given by (18) and (19). Top: The gap ratio $r_{n}$ for the $m=3$ rd band and $m=4$ th band in the $N=16, q=4$ deformed SYK model. We take the average over 1000 samples. Bottom: We consider the $m=3 \mathrm{rd}$ band in the $N=12, q=4$ deformed SYK model. The left is the gap ratio for even energy levels $r_{n}^{\prime}=\frac{E_{2 n+2}-E_{2 n}}{E_{2 n}-E_{2 n-2}}$. The right is the gap ratio $r_{n}^{\prime \prime}=\frac{E_{2 n+2}-E_{2 n+1}}{E_{2 n}-E_{2 n-1}}$. We take the average over 2000 samples for both cases.

$p(r) \sim r^{\beta}$ represents the level repulsion. We study this gap ratio numerically in the $N=16$ case and compare with the random matrix case. The $m=\frac{N}{4}=4$ th band shows GOE statistics, and the $m=3$ rd band shows GUE statistics. These result agree with our symmetry analysis above. For $m=\frac{N}{4}$ th bands in $N=4(\bmod 8)$, the degeneracies are removed at a different order of perturbation. Therefore, $E_{2 n}-E_{2 n-1}$ is of order $\mu^{-1}$, while $E_{2 n+1}-E_{2 n}$ is of order 1. In this case, we expect $E_{2 n+2}-E_{2 n}$ is determined by the first-order perturbation and looks like the GSE when $\mu$ is large. We study the gap ratio $r_{n}^{\prime}=\frac{E_{2 n+2}-E_{2 n}}{E_{2 n}-E_{2 n-2}}$ numerically, and the results show GSE statistics. On the other hand, the gap ratio $r_{n}^{\prime \prime}=\frac{E_{2 n+2}-E_{2 n+1}}{E_{2 n}-E_{2 n-1}}$ is also an order 1 quantity because both the numerator and the denominator are of order $\mu^{-1}$. These gaps $E_{2 n}-E_{2 n-1}$ come from (17), which is Hermitian. Therefore, we expect this ratio to show GUE statistics. We study this gap ratio $r_{n}^{\prime \prime}$ numerically, and it shows GUE statistics as expected.

\section{CONCLUSIONS}

The return amplitude in random matrices is exactly calculated and related to the spectral form factor. Our numerical study also shows that this relation holds in the SYK model. At early time the return amplitude decays but at late time it grows from the dip and then saturates the plateaux value. Because the random matrix behavior is expected to be universal in chaotic systems, we expect that these behaviors are true even in more generic chaotic systems like conformal field theory after a suitable average like a time average. When we deform the SYK Hamiltonian by a mass term, the return amplitude depends on the choice of initial states. If we choose the initial product states to be a ground state of the mass term, the return amplitude does not decay. This deformation prevents the initial state from being scrambled and protects from thermalization. If we flip the $m$ spins from the almost ground state one, the return amplitude decays and their behaviors are again explained by random matrix theory. The most interesting case is the $m=\frac{N}{4}$ cases in $N=4(\bmod 8)$ cases in which we see the second dip, ramp, and plateau. In early time, the return amplitude behaves like GSE statistics with two degeneracies at each level, but at late time, it realizes that the degeneracies are actually removed and finally decays to smaller values. This serves an example of the return amplitude or the spectral form factor with complicated patterns. It is interesting that, though the total deformed Hamiltonian is not time-reversal invariant, the spectrum around $E=0$ is characterized by the property of the timereversal symmetry of the SYK Hamiltonian.

\section{ACKNOWLEDGMENTS}

We thank A. Maloney, S. Harrison, and T. Takayanagi for useful discussions. We also thank R. Namba and D. Yoshida for discussions for numerical calculations. T. N. is supported by JSPS fellowships and the Simons Foundation through the It From Qubit Collaboration.

\section{APPENDIX A: EXPLICIT REALIZATION OF MAJORANA FERMIONS}

In this Appendix, we give an explicit representation of Majorana fermions. We follow the notation of Ref. [31]. We can realize the fermions as the tensor products of the Pauli matrices:

$$
\begin{aligned}
\psi_{2 k-1} & =\frac{1}{\sqrt{2}} Z_{1} \cdots Z_{k-1} X_{k} I_{k+1} \cdots I_{N / 2}, \\
\psi_{2 k} & =\frac{1}{\sqrt{2}} Z_{1} \cdots Z_{k-1} Y_{k} I_{k+1} \cdots I_{N / 2} .
\end{aligned}
$$

Here,

$X_{i}=\left(\begin{array}{ll}0 & 1 \\ 1 & 0\end{array}\right), \quad Y_{i}=\left(\begin{array}{cc}0 & -i \\ i & 0\end{array}\right), \quad Z_{i}=\left(\begin{array}{cc}1 & 0 \\ 0 & -1\end{array}\right)$

are the Pauli operators on the $i$ th site, and we omit the symbols $\otimes$ for the tensor product in (A1). Then, the $k$ th spin operator $S_{k}$ becomes

$$
S_{k}=-2 i \psi_{2 k-1} \psi_{2 k}=I_{1} \ldots I_{k-1} Z_{k} I_{k+1} \ldots I_{\frac{N}{2}} .
$$

This confirms that $S_{k}$ is the spin operator that measures the eigenvalues of $Z_{k}$. In this basis, we can write the $\left|B_{s}\right\rangle$ state as 


$$
\left|B_{s}\right\rangle=\left|s_{1} s_{2} \cdots s_{\frac{N}{2}}\right\rangle
$$

for the state with $S_{k}\left|B_{s}\right\rangle=s_{k}\left|B_{s}\right\rangle$.

We also give the explicit form of antiunitary symmetry $\mathcal{T}$ and mod 2 fermion number $(-1)^{F}$ in this basis. The fermion number $(-1)^{F}$ is given by

$$
(-1)^{F}=\prod_{k=1}^{\frac{N}{2}} S_{k}=2^{\frac{N}{2}} i^{-\frac{N}{2}} \psi_{1} \psi_{2} \cdots \psi_{N} .
$$

$\mathcal{T}$ depends on the $N$. When $N / 2$ is an odd case that corresponds to $N=2,6(\bmod 8)$,

$$
\mathcal{T}=2^{\frac{N}{4}} K \psi_{1} \psi_{3} \cdots \psi_{N-3} \psi_{N-1},
$$

where $K$ is the antiunitary operator that takes the complex conjugate. When $N / 2$ is an even case that corresponds to $N=0,4(\bmod 8)$,

$$
\mathcal{T}=2^{\frac{N}{4}} K \psi_{2} \psi_{4} \cdots \psi_{N-2} \psi_{N}
$$

Using this explicit representation, we can show the symmetry property in Table I. Though these realizations give a way to see the symmetry property, they are characterized by topological invariants [21] and independent from explicit realization.

\section{APPENDIX B: HAAR INTEGRALS AND THE DERIVATION OF THE RETURN AMPLITUDE IN RANDOM MATRICES}

We derive the return amplitude in random matrix theory. The key observation is that the measure is invariant under unitary conjugation $d\left(U^{\dagger} H U\right) e^{-V\left(U^{\dagger} H U\right)}=d H e^{-V(H)}$ with $V(H)=\frac{L}{2} \operatorname{Tr} H^{2}$. Though we choose the potential $V(H)$ of the GUE, we only need the invariance of $V(H)$ under the unitary conjugation. Then, we can represent the GUE average as $\langle f(H)\rangle_{\mathrm{GUE}}=\int d H \int d U e^{-\frac{L}{2} \operatorname{Tr} H^{2}} f\left(U^{\dagger} H U\right)$ for any function $f(H)$, where $d U$ is the Haar measure. For the return amplitude, we get

$$
\begin{aligned}
& \int d H \int d U e^{-V(H)}\left|\left\langle\psi_{0}\left|U^{\dagger} e^{-i H t} U\right| \psi_{0}\right\rangle\right|^{2} \\
& =\int d H \int d U e^{-V(H)} \operatorname{Tr}\left(e^{-i H t} U \Pi U^{\dagger} e^{i H t} U \Pi U^{\dagger}\right) .
\end{aligned}
$$

In the integrand we have the fourth moment of the unitary matrices. To evaluate this, we need the following integral:

$$
\begin{aligned}
\int & d U_{\text {Haar }} U_{i_{1} j_{1}} U_{i_{2} j_{2}} U_{i_{1}^{\prime} j_{1}^{\prime}}^{*} U_{i_{2}^{\prime} j_{2}^{\prime}}^{*} \\
= & \frac{1}{L^{2}-1}\left(\delta_{i_{1} i_{1}^{\prime}} \delta_{i_{2} i_{2}^{\prime}} \delta_{j_{1} j_{1}^{\prime}} \delta_{j_{2} j_{2}^{\prime}}+\delta_{i_{1} i_{2}^{\prime}} \delta_{i_{2} i_{1}^{\prime}} \delta_{j_{1} j_{2}^{\prime}} \delta_{j_{2} j_{1}^{\prime}}\right) \\
& -\frac{1}{L\left(L^{2}-1\right)}\left(\delta_{i_{1} i_{1}^{\prime}} \delta_{i_{2} i_{2}^{\prime}} \delta_{j_{1} j_{2}^{\prime}} \delta_{j_{2} j_{1}^{\prime}}+\delta_{i_{1} i_{2}^{\prime}} \delta_{i_{2} i_{1}} \delta_{j_{1} j_{1}^{\prime}} \delta_{j_{2} j_{2}^{\prime}}\right) .
\end{aligned}
$$

Using this integral, we obtain

$$
\left\langle\left|\left\langle\psi_{0}\left|e^{-i H t}\right| \psi_{0}\right\rangle\right|^{2}\right\rangle_{\mathrm{GUE}}=\frac{1}{L(L+1)}\left(\left\langle Z(t) Z(t)^{*}\right\rangle_{\mathrm{GUE}}+L\right) .
$$

In a similar way, we obtain

$$
\left\langle\left|\left\langle\psi_{1}\left|e^{-i H t}\right| \psi_{0}\right\rangle\right|^{2}\right\rangle_{\mathrm{GUE}}=\frac{1}{L^{2}-1}\left(L-\frac{\left\langle Z(t) Z(t)^{*}\right\rangle_{\mathrm{GUE}}}{L}\right),
$$

for orthogonal $\left|\psi_{0}\right\rangle$ and $\left|\psi_{1}\right\rangle$.

For GSE or GOE cases, the Haar integral for $U \in U(L)$ is replaced by the Haar integral for symplectic groups $S \in$ $S p(L / 2)$ or orthogonal groups $O \in O(L)$. The Haar integral for symplectic groups is given by $[27,28]$

$$
\begin{aligned}
& \int d S_{\text {Haar }} S_{i_{1} j_{1}} S_{i_{2} j_{2}} S_{i_{1}^{\prime} j_{1}^{\prime}}^{*} S_{i_{2}^{\prime} j_{2}^{\prime}}^{*} \\
& =\frac{L-1}{L(L+1)(L-2)}\left(\delta_{i_{1} i_{1}^{\prime}} \delta_{i_{2} i_{2}^{\prime}} \delta_{j_{1} j_{1}^{\prime}} \delta_{j_{2} j_{2}^{\prime}}\right. \\
& \left.\quad+\delta_{i_{1} i_{2}^{\prime}} \delta_{i_{2} i_{1}^{\prime}} \delta_{j_{1} j_{2}^{\prime}} \delta_{j_{2} j_{1}^{\prime}}+C_{i_{1} i_{2}} C_{j_{1} j_{2}} C_{i_{1}^{\prime} j_{1}^{\prime}} C_{j_{1}^{\prime} j_{2}^{\prime}}\right) \\
& \quad-\frac{1}{L(L+1)(L-2)}\left(\delta_{i_{1} i_{1}^{\prime}} \delta_{i_{2} i_{2}^{\prime}} \delta_{j_{1} j_{2}^{\prime}} \delta_{j_{2} j_{1}^{\prime}}\right. \\
& \quad+\delta_{i_{1} i_{2}^{\prime}} \delta_{i_{2} i_{1}^{\prime}} \delta_{j_{1} j_{1}^{\prime}} \delta_{j_{2} j_{2}^{\prime}}+\left(\delta_{i_{1} i_{1}^{\prime}} \delta_{i_{2} i_{2}^{\prime}}-\delta_{i_{1} i_{2}^{\prime}} \delta_{i_{2} i_{1}^{\prime}}\right) C_{j_{1} j_{2}} C_{j_{1}^{\prime} j_{2}^{\prime}} \\
& \left.\quad+C_{i_{1} i_{2}} C_{i_{1}^{\prime} i_{2}^{\prime}}\left(\delta_{j_{1} j_{1}^{\prime}} \delta_{j_{2} j_{2}^{\prime}}-\delta_{j_{1} j_{2}^{\prime}} \delta_{j_{2} j_{1}^{\prime}}\right)\right),
\end{aligned}
$$

where $C_{i j}$ is the antisymmetric invariant. This coupling gives the inner product between the Kramers pairs. Because this coupling is antisymmetric, the diagonal part $v_{i} C_{i j} v_{j}$ for $\left|\psi_{0}\right\rangle=\sum_{i} v_{i}\left|e_{i}\right\rangle$ vanishes, and they can be ignored in the calculation of the return amplitude. Using this integral, we obtain

$$
\left\langle\left|\left\langle\psi_{0}\left|e^{-i H t}\right| \psi_{0}\right\rangle\right|^{2}\right\rangle_{\mathrm{GSE}}=\frac{1}{L(L+1)}\left(\left\langle Z(t) Z(t)^{*}\right\rangle_{\mathrm{GSE}}+L\right),
$$

which is the same expression with the GUE case, though the Haar integrals themselves are different.

The Haar integral for orthogonal groups is given by $[27,28]$ 


$$
\begin{aligned}
& \int d O_{\text {Haar }} O_{i_{1} j_{1}} O_{i_{2} j_{2}} O_{i_{1}^{\prime} j_{1}^{\prime}}^{*} O_{i_{2}^{\prime} j_{2}^{\prime}}^{*} \\
& =\frac{L+1}{L(L-1)(L+2)}\left(\delta_{i_{1} i_{1}^{\prime}} \delta_{i_{2} i_{2}^{\prime}} \delta_{j_{1} j_{1}^{\prime}} \delta_{j_{2} j_{2}^{\prime}}\right. \\
& \left.\quad+\delta_{i_{1} i_{2}^{\prime}} \delta_{i_{2} i_{1}^{\prime}} \delta_{j_{1} j_{2}^{\prime}} \delta_{j_{2} j_{1}^{\prime}}+C_{i_{1} i_{2}} C_{j_{1} j_{2}} C_{i_{1}^{\prime} j_{1}^{\prime}} C_{j_{1}^{\prime} j_{2}^{\prime}}\right) \\
& \quad-\frac{1}{L(L-1)(L+2)}\left(\delta_{i_{1} i_{1}^{\prime}} \delta_{i_{2} i_{2}^{\prime}} \delta_{j_{1} j_{2}^{\prime}} \delta_{j_{2} j_{1}^{\prime}}\right. \\
& \quad+\delta_{i_{1} i_{2}^{\prime}} \delta_{i_{2} i_{1}^{\prime}} \delta_{j_{1} j_{1}^{\prime}} \delta_{j_{2} j_{2}^{\prime}}+\left(\delta_{i_{1} i_{1}^{\prime}} \delta_{i_{2} i_{2}^{\prime}}+\delta_{i_{1} i_{2}^{\prime}} \delta_{i_{2} i_{1}^{\prime}}\right) C_{j_{1} j_{2}} C_{j_{1}^{\prime} j_{2}^{\prime}} \\
& \left.\quad+C_{i_{1} i_{2}} C_{i_{1}^{\prime} i_{2}^{\prime}}\left(\delta_{j_{1} j_{1}^{\prime}} \delta_{j_{2} j_{2}^{\prime}}+\delta_{j_{1} j_{2}^{\prime}} \delta_{j_{2} j_{1}^{\prime}}\right)\right),
\end{aligned}
$$

where $C_{i j}$ is the symmetric coupling and we can choose a basis with $C_{i j}=\delta_{i j}$. Unlike the case of symplectic groups, the diagonal part $v_{i} C_{i j} v_{j}$ for $\left|\psi_{0}\right\rangle=\sum_{i} v_{i}\left|e_{i}\right\rangle$ does not vanish, and the return amplitude depends on states.

\section{APPENDIX C: SYMMETRY ANALYSIS OF THE DEFORMED SYK HAMILTONIAN AT FIRST-ORDER PERTURBATION}

In this Appendix, we study the symmetry class $H_{m}$ in detail that is imposed by $\mathcal{T}$ symmetry. Because $\mathcal{T}$ relates the $m$ th band and the $\left(\frac{N}{2}-m\right)$ th band, it is sufficient to consider the symmetry constraint on the submatrix

$$
H^{m, \frac{N}{2}-m}=\left(\begin{array}{cc}
H_{m} & S_{m} \\
S_{m}^{\dagger} & H_{\frac{N}{2}-m}
\end{array}\right),
$$

where $H_{m}=P_{m} H_{\mathrm{SYK}} P_{m}$ and $S_{m}=P_{m} H_{\mathrm{SYK}} P_{\frac{N}{2}-m}$. Using the symmetry of the SYK Hamiltonian $(-1)^{F} H_{\mathrm{SYK}}(-1)^{F}=$ $H_{\mathrm{SYK}}$, we find $P_{m} H_{\mathrm{SYK}} P_{\frac{N}{2}-m}=(-1)^{\frac{N}{2}} P_{m} H_{\mathrm{SYK}} P_{\frac{N}{2}-m}$. This means $S_{m}=0$ for $N=2,6(\bmod 8)$. For $N=0,2(\bmod 8)$ cases, $\mathcal{T}^{2}=1$. In these cases, we can choose the basis with $\mathcal{T}=\left(\begin{array}{cc}0 & \mathbb{I}_{d_{m}} \\ \mathbb{I}_{d_{m}} & 0\end{array}\right) K$, where $\mathbb{I}_{d_{m}}$ is the identity matrix of rank $d_{m}$ and $K$ is the complex conjugate operator. The invariance under this $\mathcal{T}$ imposes the condition $H_{\frac{N}{2}-m}^{*}=H_{m}$ and $S_{m}^{T}=S_{m}$. For $N=0(\bmod 8)$, this means that $H^{m, \frac{N}{2}-m}$ is conjugate to real symmetric matrix, though $H^{m, \frac{N}{2}-m}$ is expressed in an unusual basis in which the reality is not manifest. To see the reality manifestly, it is convenient to change the basis in the following way:

$$
\begin{aligned}
\left(\begin{array}{cc}
H_{m} & S_{m} \\
S_{m}^{*} & H_{m}^{*}
\end{array}\right) & \rightarrow \frac{1}{\sqrt{2}}\left(\begin{array}{cc}
\mathbb{I}_{d_{m}} & \mathbb{I}_{d_{m}} \\
i \mathbb{I}_{d_{m}} & -i \mathbb{I}_{d_{m}}
\end{array}\right)\left(\begin{array}{cc}
H_{m} & S_{m} \\
S_{m}^{*} & H_{m}^{*}
\end{array}\right) \\
& \times \frac{1}{\sqrt{2}}\left(\begin{array}{cc}
\mathbb{I}_{d_{m}} & -i \mathbb{I}_{d_{m}} \\
\mathbb{I}_{d_{m}} & i \mathbb{I}_{d_{m}}
\end{array}\right) \\
= & \left(\begin{array}{cc}
\operatorname{Re} H_{m}+\operatorname{Re} S_{m} & \operatorname{Im} H_{m}-\operatorname{Im} S_{m} \\
-\operatorname{Im} H_{m}-\operatorname{Im} S_{m} & \operatorname{Re} H_{m}-\operatorname{Re} S_{m}
\end{array}\right) .
\end{aligned}
$$

This takes the form of the real symmetric matrix under the condition $S_{m}^{T}=S_{m}$ and $H_{m}^{\dagger}=H_{m}$. Though $\mathcal{T}$ relates $H_{m}$ and $H_{\frac{N}{2}-m}$, it does not impose any constraint on $H_{m}$ itself. Therefore, for generic matrix $H^{m, \frac{N}{2}-m}, H_{m}$ belongs to the GUE.

For $N=4,6(\bmod 8), \mathcal{T}^{2}=-1$. In these cases, we can choose the basis with $\mathcal{T}=\left(\begin{array}{cc}0 & -\mathbb{I}_{d_{m}} \\ \mathbb{I}_{d_{m}} & 0\end{array}\right) K$. In the same manner, we obtain $H_{m}=H_{\frac{N}{2}-m}^{*}$ and $S_{m}^{T}=-S_{m}$. For $N=4(\bmod 8)$, this means that $H^{m, \frac{N}{2}-m}$ is a quaternion Hermitian. We can write it as $H_{m}=H+i A_{z}$ and $S_{m}=i A_{x}+A_{y}$, where $H$ is real symmetric and $A_{x}, A_{y}, A_{z}$ are real antisymmetric. Then, $H^{m, \frac{N}{2}-m}=H \otimes \mathbb{I}_{2}+\sum_{\alpha} i A_{\alpha} \otimes \sigma_{\alpha}$ gives an usual realization of quaternion Hermitian matrices. $H_{m}=H+i A_{z}$ spans generic Hermitian matrices, and the ensemble is GUE. Again, though $\mathcal{T}$ relates $H_{m}$ and $H_{\frac{N}{2}-m}, \mathcal{T}$ does not impose any condition on $H_{m}$ itself.

\section{APPENDIX D: TIME AVERAGE OF SINGLE SAMPLE IN THE SYK MODEL}

The spectral form factor is not self-averaging [32], and we need some averages to get smooth behaviors. In the SYK model, we take the ensemble average over the coupling $J_{a_{1} \cdots a_{q}}$. In this Appendix, we consider the time average of the return amplitude in a single sample in the SYK model, which is another kind of average. First, we consider the infinite time average, which gives the averaged plateau value, in general quantum systems without degeneracy. By decomposing the state $\left|\psi_{0}\right\rangle=\sum_{i} c_{i}\left|E_{i}\right\rangle$, we obtain

$$
\begin{aligned}
& \lim _{T \rightarrow \infty} \frac{1}{T} \int_{0}^{T}\left|\left\langle\psi_{0}\left|e^{-i H t}\right| \psi_{0}\right\rangle\right|^{2} \\
& =\lim _{T \rightarrow \infty} \frac{1}{T} \int_{0}^{T} d t \sum_{i, j} c_{i}^{*} c_{i} c_{j}^{*} c_{j} e^{-i\left(E_{i}-E_{j}\right) t} \\
& =\sum_{i}\left|c_{i}\right|^{4} .
\end{aligned}
$$

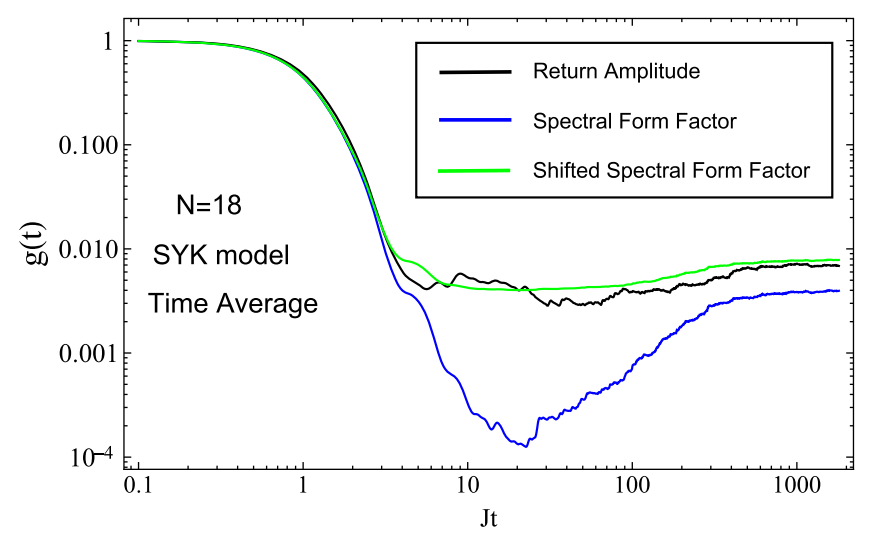

FIG. 6. This is the plot of the return amplitude in the $N=18$,

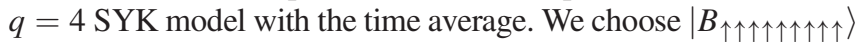
as the initial state. We can see the slope, dip, ramp, and plateau even in the time-average cases. As we mentioned, the plateau value of the time averaged return amplitude has a small deviation from the shifted spectral form factor. 
Therefore, the time average is not exactly the same with the plateau value in the ensemble average (3). If we take the average of (D1) over states, it becomes the late time value $2 /(L+1)$ in the ensemble average. In the SYK model, we consider the following time average:

$$
g_{p}^{\mathrm{time}}(t ; \beta)=\int_{\frac{1}{2} t}^{\frac{3}{2} t}\left|\left\langle B_{s}\left|e^{-i H_{\mathrm{SYK}} t^{\prime}-\beta H_{\mathrm{SYK}}}\right| B_{s}\right\rangle\right|^{2} d t^{\prime} .
$$

Here, we take the time average between $\frac{1}{2} t<t^{\prime}<\frac{3}{2} t$ around time $t$, which is taken in Ref. [23]. We show the plots of the return amplitude and the spectral form factor with time average in Fig. 6. Both the return amplitude and the spectral form factor show the slope, dip, ramp, and plateau behavior. This motivates us to expect that the return amplitude in more generic quantum systems like chaotic conformal field theories shows these structures after the time average. We also compare the shifted spectral form factor (D1) in which the ensemble average of the spectral form factor is replaced by the time average. As we pointed out, their late time value is not exactly the same, but the behavior shows good agreement on each time.
[1] S. Sachdev and J. Ye, Phys. Rev. Lett. 70, 3339 (1993).

[2] A. Kitaev, A Simple Model of Quantum Holography, KITP Strings Seminar and Entanglement 2015 Program (2015).

[3] J. Maldacena and D. Stanford, Phys. Rev. D 94, 106002 (2016).

[4] A. Kitaev and S. J. Suh, J. High Energy Phys. 05 (2018) 183.

[5] J. Maldacena, S. H. Shenker, and D. Stanford, J. High Energy Phys. 08 (2016) 106.

[6] Y.-Z. You, A. W. W. Ludwig, and C. Xu, Phys. Rev. B 95, 115150 (2017).

[7] J. S. Cotler, G. Gur-Ari, M. Hanada, J. Polchinski, P. Saad, S. H. Shenker, D. Stanford, A. Streicher, and M. Tezuka, J. High Energy Phys. 05 (2017) 118; 09 (2018) 002(E).

[8] R. Jackiw, Nucl. Phys. B252, 343 (1985).

[9] C. Teitelboim, Phys. Lett. 126B, 41 (1983).

[10] A. Almheiri and J. Polchinski, J. High Energy Phys. 11 (2015) 014.

[11] J. Maldacena, D. Stanford, and Z. Yang, Prog. Theor. Exp. Phys. 2016, 12C104 (2016).

[12] I. Kourkoulou and J. Maldacena, arXiv:1707.02325.

[13] C. Krishnan and K. V. P. Kumar, J. High Energy Phys. 10 (2017) 099.

[14] N. Hunter-Jones, J. Liu, and Y. Zhou, J. High Energy Phys. 02 (2018) 142.

[15] A. Dhar, A. Gaikwad, L. K. Joshi, G. Mandal, and S. R. Wadia, arXiv:1812.03979.

[16] R. Bhattacharya, D. P. Jatkar, and N. Sorokhaibam, J. High Energy Phys. 07 (2019) 066.

[17] T. Numasawa, N. Shiba, T. Takayanagi, and K. Watanabe, J. High Energy Phys. 08 (2016) 077.

[18] J. Cardy, Phys. Rev. Lett. 112, 220401 (2014).

[19] J. Cardy, J. Phys. A 49, 415401 (2016).

[20] R. Brustein and Y. Zigdon Phys. Rev. D 98, 066013 (2018).

[21] L. Fidkowski and A. Kitaev, Phys. Rev. B 83, 075103 (2011).
[22] T. Kanazawa and T. Wettig J. High Energy Phys. 09 (2017) 050.

[23] V. Balasubramanian, B. Craps, B. Czech, and G. Srosi, J. High Energy Phys. 03 (2017) 154.

[24] N. Benjamin, E. Dyer, A. L. Fitzpatrick, and Y. Xin, J. High Energy Phys. 04 (2019) 025.

[25] A. Chenu, J. Molina-Vilaplana, and A. Del Campo, Quantum 3, 127 (2019).

[26] A. del Campo, J. Molina-Vilaplana, and J. Sonner, Phys. Rev. D 95, 126008 (2017).

[27] B. Collins and P. Śniady, Commun. Math. Phys. 264, 773 (2006).

[28] A. Altland and M. R. Zirnbauer, Phys. Rev. B 55, 1142 (1997).

[29] M. Schiulaz, E. J. Torres-Herrera, and L. F. Santos, Phys. Rev. B 99, 174313 (2019).

[30] J. Cotler, N. Hunter-Jones, J. Liu, and B. Yoshida J. High Energy Phys. 11 (2017) 048.

[31] P. Saad, S. H. Shenker, and D. Stanford, arXiv:1806.06840.

[32] R. E. Prange, Phys. Rev. Lett. 78, 2280 (1997).

[33] A. Eberlein, V. Kasper, S. Sachdev, and J. Steinberg, Phys. Rev. 96B, 205123 (2017).

[34] A. M. Garca-Garca, B. Loureiro, A. Romero-Bermdez, and M. Tezuka Phys. Rev. Lett. 120, 241603 (2018).

[35] T. Nosaka, D. Rosa, and J. Yoon, J. High Energy Phys. 09 (2018) 041.

[36] A. Messiah, Quantum Mechanics, Dover Books on Physics (Dover, New York, 1999).

[37] D. J. Luitz, N. Laflorencie, and F. Alet, Phys. Rev. B 91, 081103 (2015).

[38] V. Oganesyan and D. A. Huse, Phys. Rev. B 75, 155111 (2007).

[39] C. L. Bertrand and A. M. García-García, Phys. Rev. B 94, 144201 (2016).

[40] Y. Y. Atas, E. Bogomolny, O. Giraud, and G. Roux, Phys. Rev. Lett. 110, 084101 (2013). 